\title{
Axiomatic design and virtual verification for blackbody cavity sensor
}

\author{
Jiangfeng Cheng ${ }^{1,2}$, Chia-Feng Juang, ${ }^{2, *}$, Weihai Chen ${ }^{1}$, and Guohui Mei ${ }^{3, *}$ \\ ${ }^{1}$ School of Automation Science and Electrical Engineering, Beihang University, Beijing 100191, China \\ ${ }^{2}$ Department of Electrical Engineering, National Chung Hsing University, Taichung 402, TW \\ ${ }^{3}$ College of Information Science and Engineering, Northeastern University, Shenyang 110819, China
}

\begin{abstract}
The blackbody cavity sensor for continuous temperature measurement of molten steel has been widely used in steel industry. However, due to the closed bottom of the inner tube, the temperature measurement accuracy is seriously affected. It's urgent to redesign and improve the sensor, which involves multidisciplinary knowledge, including materials, heat and flow science. This paper first clarifies the relationship between sensor functional requirements and various physical structure parameters from the perspective of axiomatic design. On this basis, the virtual models of the blackbody cavity sensor are established, including geometry model, multi-physical field model, material physical properties and boundary conditions. And then through comparison between experiment and simulation, it is found that for the temperature measurement accuracy, the deviations between the simulation and the actual experimental result are less than $1.5^{\circ} \mathrm{C}$. This verifies the accuracy of the virtual model.
\end{abstract}

\section{Introduction}

The blackbody cavity sensor for continuous temperature measurement of molten steel, which consists of a cylindrical outer tube with a flat bottom, a coaxial inner tube, and an aperture diaphragm, is being widely used in steel industry, as shown in Fig. 1(a) [1-2]. The sensor adopts a coaxial casing structure. The outer tube is made of refractory material, which blocks the erosion of molten steel. The inner tube is a corundum tube closed at the bottom end, which creates a clean temperature measuring cavity [3].

However, due to the closed semicircle at the bottom of the corundum tube, inner corundum tube of the sensor is prone to bending and even cracking under the high temperature. This causes that temperature

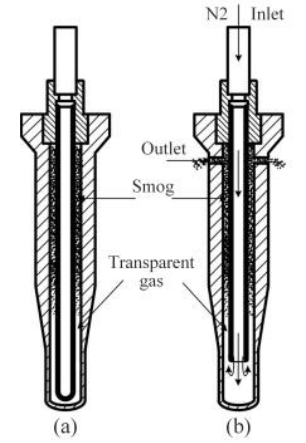

Fig. 1. Blackbody cavity temperature sensor. measurement value of the sensor is easily interfered, and the sensor has a short life.

To improve the performance of the sensor, reduce manufacturing cost and improve service life, a new design scheme is proposed, as shown in Fig. 1(b). The scheme removes the semicircular bottom end of the inner corundum tube and appropriately shortens its length.

But, the outer tube will evaporate the impurity gas under the high temperature, and the main component of the impurity is sodium oxide. When the temperature is higher than $1275^{\circ} \mathrm{C}$, it is a transparent gas, which does not affect the temperature measurement. However, when the temperature is lower than $1275^{\circ} \mathrm{C}$, the impurity gas will aggregate into particles [4]. The particles block the light path and cause the radiation intensity received by the temperature sensor to decrease, thereby affecting the temperature measurement result. To further solve the problem, it is proposed to blow nitrogen gas into the cavity from the top of the corundum tube, and drill two vent holes on upper part of the outer tube to achieve the purpose of cleaning the light path. However, under different nitrogen flow rates and inner tube lengths, the temperature measurement value and the temperature measurement stability time will be different.

In order to clarify the relationship between sensor functional requirements and various physical structure parameters, this research first analyzes the sensor design improvement strategy and method from the perspective of axiomatic design [5-7]. Mapping relationship is established between functional requirements including thermal shock problem of inner tube, inner tube bending degree and temperature measurement accuracy, and design parameters including whether bottom of the inner tube is closed, inner tube length, and nitrogen flow rate. On this basis, the research focus is clarified, that is, finding out the relationship between temperature measurement accuracy and inner tube length, nitrogen flow rate. This paper establishes virtual model of the blackbody cavity sensor, as shown in Section III. Simulation analysis and actual experiment are carried out next, as shown in Section IV. The virtual model is iteratively modified through considering the influence of

\footnotetext{
* Corresponding author: Chia-Feng Juang (ㄷjuang@dragon.nchu.edu.tw); Guohui Mei (meiguohui@ise.neu.edu.cn)
} 
different nitrogen flow rates and different inner tube's lengths on temperature measurement accuracy.

\section{Axiomatic design method for blackbody cavity sensor}

Firstly, it is necessary to determine the functional requirements, specifically what functional requirements are included, and the design parameters, specifically including the physical structure and structural parameters. Mapping relationship between functional requirements and physical parameters needs to be established. On this basis, the relationship is decoupled to get the optimal design method with the minimal information content of the design [8-10].

There are three main functional requirements, including thermal shock performance of inner corundum tube (bottom of the inner tube is closed, resulting in thermal stress concentration and being fragile), bending degree of inner tube (which affects the perception of the temperature radiation surface) and temperature deviation of measured value from actual true value. They can be expressed as $F R_{1}, F R_{2}$ and $F R_{3}$, respectively.

The relevant design parameters for physical structure of the sensor mainly include: $D P_{1}$, whether bottom of the inner tube is closed, $D P_{2}$, length of the inner tube, and $D P_{3}$, nitrogen flow rate blown into the sensor.

Since vent hole of the sensor is located at the upper part of the outer Al-carbon tube as shown in Fig. 1, according to the blackbody cavity radiation temperature measurement theory [3]. Position and number of the vent hole do not affect temperature measurement accuracy $\left(F R_{3}\right)$. In addition, position and number of the vent holes do not affect the thermal shock performance of inner tube $\left(F R_{1}\right)$ and its bending degree $\left(F R_{2}\right)$. Therefore, the two physical parameters do not affect the functional requirements and are not considered.

The mapping relationship $\{\mathrm{FR}\}=[\mathrm{A}]\{\mathrm{DP}\}$ between functional requirements and design parameters is established, as shown below, where [A] is the design matrix.

$$
\left[\begin{array}{l}
F R_{1} \\
F R_{2} \\
F R_{3}
\end{array}\right]=\left[\begin{array}{ccc}
A_{11} & 0 & 0 \\
A_{21} & A_{22} & 0 \\
A_{31} & A_{32} & A_{33}
\end{array}\right]\left[\begin{array}{c}
D P_{1} \\
D P_{2} \\
D P_{3}
\end{array}\right]
$$

According to the axiomatic design theorem, the matrix [A] needs to be decoupled to meet the independent axiom of functional requirements [5-6]. The design parameter of whether bottom of the inner tube is closed, is coupled with the three main functional requirements. Considering the short life or even instability due to the thermal stress concentration, the closed bottom of inner tube must be removed in the improvement scheme. As a result, the thermal shock problem of inner corundum tube is solved, the mapping relationship can be further decoupled as follows:

$$
\left[\begin{array}{l}
F R_{2} \\
F R_{3}
\end{array}\right]=\left[\begin{array}{cc}
A_{22} & 0 \\
A_{32} & A_{33}
\end{array}\right]\left[\begin{array}{l}
D P_{2} \\
D P_{3}
\end{array}\right]
$$

Further, considering that the above design matrix is still a coupling matrix, in real-world application, it can be found that open-ended inner corundum tubes with different lengths have little effect on their bending degree, and do not affect the temperature measurement accuracy at all. Therefore, the above mapping relationship can be further decoupled as follows:

$$
\left.\left[\begin{array}{ll}
F R_{3}
\end{array}\right]=\left[\begin{array}{ll}
A_{32} & A_{33}
\end{array}\right] \begin{array}{l}
D P_{2} \\
D P_{3}
\end{array}\right]
$$

In other words, the sensor temperature measurement accuracy that the user is most concerned about is related to the length of inner corundum tube and the nitrogen flow rate. Therefore, a coupled parallel design process with inner corundum tube length and nitrogen flow rate is required with simulation and experiment method [11].

To make sense of the influence mechanism of different nitrogen flow rates and inner tube lengths on temperature measurement accuracy, this paper conducts research from two steps.

Step 1: virtual model establishment. First, the virtual model of blackbody cavity sensor for continuous temperature measurement of molten steel needs to be established, which has high fidelity with physical one and can truly reflect its behaviour, rule, constraint, etc. The virtual model should have the following two characteristics. (1) Physical-virtual synchronization and consistency characteristics. It guarantees the accuracy of virtual model construction. (2) The integrated multiphysics, multiscale, probabilistic high-fidelity simulation capability. It provides powerful simulation and analysis capability for virtual simulation. However, the modelling process involves temperature field including heat transfer, convection heat transfer, radiation heat transfer and heat transfer in soot phase change, and flow field including turbulent diffusion, multiphase flow and Buoyancydriven flow. Thus the process is more complicated.

Step 2: virtual model verification. It is another extremely important part to verify the accuracy of the established virtual model, so as to iteratively optimize and gradually modify the virtual model [12-13]. Virtual verification, on one hand, can provide theoretical support for the design improvement. This makes it easier to upgrade and maintain the subsequent product. On the other hand, it can also improve product performance and service quality, and hence to reduce production cost and maintenance cycle. Only if the virtual model of blackbody cavity sensor is verified, it is possible to carry out simulation experiments under different nitrogen flow rates and different inner tube lengths, and then conduct design improvement of blackbody cavity sensor.

\section{Virtual model for blackbody cavity sensor}

Regardless of how to change the structure of the blackbody cavity sensor and how to improve its performance, the ultimate goal is still to ensure the accuracy of temperature measurement. After the sensor is blown with nitrogen for a certain period of time in actual 
use, the soot impurity is blown out of the cavity. The cavity inside is clean, so that the light path is not blocked. In terms of the consequences, it is only the temperature distribution of the cavity that is affected by the different nitrogen flow rates. Therefore, to reduce the research complexity, in the early stage of the research, only the temperature field and flow field involved in the final steady state are considered while constructing the virtual model. Issues such as multiphase flow, phase change and particle radiation involved in transient conditions are not considered. While verifying the established virtual model, only the influence of different nitrogen flow rates on the final temperature measurement accuracy is considered, instead of temperature measurement stability time.

\subsection{Geometry model}

The sensor is inserted vertically into molten steel during use, as shown in Fig. 2(a). Nitrogen gas is blown in from the top fixing tube, blown downward from the cavity of the inner tube, blown upward from the outer side of the inner tube, and finally blown out from the exhaust holes at the upper of the outer tube.

While establishing geometry model, the portion of the fixed tube and the outer tube above the vent holes are omitted. The geometry model, as shown in Fig. 2(b), consists of three parts, i.e., an outer Al-carbon tube, an inner corundum tube, and an internal cavity formed by the two tubes.

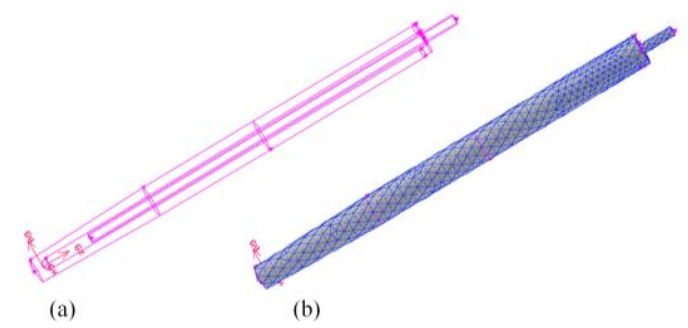

Fig. 2. Geometry model of blackbody cavity sensor.

Then the constructed geometry model needs to be meshed. The region from the bottom of corundum tube to the bottom of the outer tube has the greatest influence on the temperature measurement value. Therefore, the mesh is dense here and meshed by the mesh size 2, as shown in Fig. 2(c). After checking, the EquiSize Skew of more than $90 \%$ of the meshes is below 0.5 . There is no mesh with EquiSize Skew greater than 0.8. Therefore, it can be said that the mesh quality is relatively good, which can meet the subsequent calculation requirements.

\subsection{Multi-physical field model}

\subsubsection{Heat conduction and convection heat transfer model}

(1) Heat transfer and convection heat transfer model for fluid regions and fluid-solid regions

This includes energy transfer from nitrogen flow, and energy transfer between the nitrogen and solid regions. [14]

$$
\frac{\partial}{\partial t}(\rho E)+\nabla \cdot(\vec{v} \rho E)=\nabla \cdot\left(k_{e f f} \nabla T-\sum h_{j} \vec{J}_{j}\right)
$$

where $k_{\text {eff }}$ is the effective thermal conductivity, and $\vec{J}_{j}$ is the diffusion flux of component $j$. In this equation, $k_{e f f} \nabla T$ and $\sum h_{j} \vec{J}_{j}$ represent the energy transfer caused by heat conduction and component diffusion, respectively. $E$ represents the total energy of the fluid micelle, i.e., the sum of internal energy and kinetic energy.

$$
E=\sum_{j} Y_{j} \int_{T_{r e f}}^{T} c_{p, j} d T+\frac{v^{2}}{2}
$$

where $Y_{j}$ is the mass fraction of component $j$, and the reference temperature value $T_{\text {ref }}$ is $298.15 \mathrm{~K}$.

For convection heat transfer, the Buoyancy driving flow needs to be considered. The density of the substance needs to be set to a temperature-dependent function $\rho=$ $f(T)$. The density function expression of nitrogen can be found in literature [15-16].

\section{(2) Heat transfer model for solid regions}

The outer wall of the Al-carbon tube under molten steel and the wall of the inner corundum tube have heat sources $S_{h}$, and the heat transfer model is as follows [14]:

$$
\frac{\partial}{\partial t}(\rho h)=\nabla \cdot(k \nabla T)+S_{h}
$$

There is no heat source in other parts of the solid region, and the heat transfer model is as follows:

$$
\frac{\partial}{\partial t}(\rho h)=\nabla \cdot(k \nabla T)
$$

The thermal conductivity of the inner and outer tubes in the composite cavity can be regarded as isotropic. Therefore, the corresponding thermal conductivity is set to be the scalar constant.

\subsubsection{Radiation heat transfer model}

The sensor is used in a high temperature environment of around $1800 \mathrm{~K}$, and radiation heat transfer plays an extremely important role. In the establishment of the radiation model, on the one hand, the limitation of optical thickness is considered. On the other hand, it needs to consider the scattering of soot particles/vapor and the effect of soot particles on radiation for the subsequent transient modelling. Therefore, Discrete Ordinates (DO) radiation model is adopted.

Gray-band radiation model is used to simulate nongray radiation. Based on the spectral intensity $I_{\lambda}(\vec{r}, \vec{s})$, the radiation transfer equation is as follows [17-18]:

$$
\begin{gathered}
\nabla \cdot\left(I_{\lambda}(\vec{r}, \vec{s}) \vec{s}\right)+\left(a+\sigma_{s}\right) I_{\lambda}(\vec{r}, \vec{s})=a_{\lambda} n^{2} I_{\mathrm{b} \lambda}+ \\
\frac{\sigma_{s}}{4 \pi} \int_{0}^{4 \pi} I_{\lambda}\left(\vec{r}, \vec{s}^{\prime}\right) \Phi\left(\vec{s}, \vec{s}^{\prime}\right) d \Omega^{\prime}
\end{gathered}
$$

where

$\begin{array}{ll}\vec{r}=\text { position vector } & \Phi=\text { phase function } \\ \vec{S}=\text { direction vector } & \Omega^{\prime}=\text { solid angle } \\ \vec{S}^{\prime}=\text { scattering direction } & \left(a+\sigma_{s}\right) s=\text { medium's } \\ \quad \text { vector } & \text { optical thickness } \\ S=\text { path length } & \lambda=\text { radiation wavelength }\end{array}$




$$
\begin{array}{cc}
\begin{array}{c}
a=\text { absorption } \\
\text { coefficient }
\end{array} & a_{\lambda}=\text { spectral absorption } \\
& \text { coefficient } \\
n=\text { refractive index } & I_{\mathrm{b} \lambda}=\text { blackbody radiation } \\
& \text { intensity } \\
\sigma_{s}=\text { scattering } & \sigma=\text { Stefan-Boltzmann } \\
\text { coefficient } & \text { constant }(5.672 \times \\
I=\text { radiation intensity } & \left.10^{-8} \mathrm{~W} / \mathrm{m}^{2}-K^{4}\right)
\end{array}
$$

The scattering coefficient, scattering phase function, and refractive index are all considered to be wavelength independent.

The non-gray DO radiation model divides the entire radiation spectral band into $N$ bands, integrates the wavelengths over all wavelength ranges, thus obtaining a transport equation about $I_{\lambda} \Delta \lambda$. Radiation heat transfer is contained within each band $\Delta \lambda$. Within each band, it is considered to be blackbody radiation, and its blackbody radiation in unit solid angle is as the equation (7).

$$
\left[F\left(0 \rightarrow n \lambda_{2} T\right)-F\left(0 \rightarrow n \lambda_{1} T\right)\right] n^{2} \frac{\sigma T^{4}}{\pi}
$$

where $F(0 \rightarrow n \lambda T)$ is the blackbody radiation in the range of $0 \sim \lambda$ wavelength at the temperature $T$ under the medium of refractive index $n$, and $\lambda_{1}, \lambda_{2}$ are the boundary of the band.

The blackbody cavity sensor in this research belongs to full-wave radiation. At any temperature in the cavity, the ratio of the radiation intensity of all wavelengths to the radiation intensity of the absolute blackbody at the corresponding wavelength is fixed. Therefore, it is regarded as gray radiation, that is, the emissivity is constant.

The walls of the outer Al-carbon tube adjacent to the fluid region and the inner corundum tube belong to the opaque wall surface. When the radiation of the fluid region hits the wall of the inner cavity, specular reflection and diffuse reflection will occur. The wall absorbs some of the energy, while also radiates energy outward, which is related to the emissivity. Fig. 3 shows the radiation mechanism of the inner wall surface of the sensor.

The expressions of wall emission energy, diffuse reflected energy, specular reflection energy and absorbed radiant energy in Fig. 3 are given as follows. [19-20]



Fig. 3. Radiation mechanism of the inner wall of the sensor.

$$
\begin{gathered}
q_{e}=n^{2} \varepsilon_{w} \sigma T_{w}^{4} \\
q_{d r}=f_{d}\left(1-\varepsilon_{w}\right) q_{i n} \\
q_{s r}=\left(1-f_{d}\right)\left(1-\varepsilon_{w}\right) q_{i n} \\
q_{a}=\varepsilon_{w} q_{i n}
\end{gathered}
$$

where $f_{d}$ is the diffusion coefficient, $n$ is the refractive index of the fluid in the cavity, $\varepsilon_{w}$ is the wall emissivity, and $T_{w}$ is the wall temperature. The incident radiant energy $q_{\text {in }}$ of the inner wall of the cavity is

$$
q_{\text {in }}=\int_{\vec{s} \cdot \vec{n}>0} I_{i n} \vec{s} \cdot \vec{n} d \Omega
$$

The net radiant energy $q_{\text {out }}$ leaving the wall is

$$
q_{\text {out }}=\left(1-\varepsilon_{w}\right) q_{\text {in }}+n^{2} \varepsilon_{w} \sigma T_{w}^{4}
$$

For all $\vec{s}$ leaving the wall, the radiation intensity of the wall is as follows:

$$
I_{o}=\frac{q_{o u t}}{\pi}
$$

\subsubsection{Turbulence model}

In this paper, the nitrogen flow rates are set to 0.25 $2.5 \mathrm{~m}^{3} / \mathrm{h}$. The Reynolds number is between $1000 \sim 4000$. Moreover, due to the complex geometry, the fluid flow in the cavity is considered to be a fully developed turbulence.

In the cavity of the sensor, there is no vortex, rotation and rapid change of tension during the blowing process of soot under the forced convection. The RNG $k-\varepsilon$ model has a wide range of applications and is economical and reasonable. The model has undergone practical tests of numerous engineering fluid problems and is relatively reliable. Therefore, this model will be used in this research, as shown below. [21-23]

$$
\begin{gathered}
\frac{\partial}{\partial t}(\rho k)+\frac{\partial}{\partial x_{i}}\left(\rho k u_{i}\right)=\frac{\partial}{\partial x_{j}}\left(\alpha_{k} \mu_{e f f} \frac{\partial k}{\partial x_{j}}\right) \\
+G_{k}+G_{b}-\rho \varepsilon+S_{k} \\
\frac{\partial}{\partial t}(\rho \varepsilon)+\frac{\partial}{\partial x_{i}}\left(\rho \varepsilon u_{i}\right)=\frac{\partial}{\partial x_{j}}\left(\alpha_{\varepsilon} \mu_{e f f} \frac{\partial \varepsilon}{\partial x_{j}}\right) \\
+C_{1 \varepsilon} \frac{\varepsilon}{k}\left(G_{k}+C_{3 \varepsilon} G_{b}\right)-C_{2 \varepsilon}^{*} \rho \frac{\varepsilon^{2}}{k}+S_{\varepsilon}
\end{gathered}
$$

where $\alpha_{k}$ and $\alpha_{\varepsilon}$ are the turbulent Prandtl numbers of the $k$ and $\varepsilon$ equations, respectively, which are defined in equation (17), where $\alpha_{0}=1.0$

$$
\left|\frac{\alpha-1.3929}{\alpha_{0}-1.3929}\right|^{0.6321}\left|\frac{\alpha+2.3929}{\alpha_{0}+2.3929}\right|^{0.3679}=\frac{\mu_{m o l}}{\mu_{e f f}}
$$

and $C_{1 \varepsilon}=1.42, C_{2 \varepsilon}=1.68$, and the expression of $C_{2 \varepsilon}^{*}$ is as follows:

$$
C_{2 \varepsilon}^{*} \equiv C_{2 \varepsilon}+\frac{C_{\mu} \eta^{3}\left(1-{ }^{\eta} / \eta_{0}\right)}{1+\beta \eta^{3}}
$$

and $G_{k}$ is the turbulence kinetic energy generated by the mean velocity gradients which is given by

$$
G_{k}=-\rho \overline{u_{\imath}^{\prime} u_{\jmath}^{\prime}} \frac{\partial u_{j}}{\partial x_{i}}
$$

The established energy exchange model needs to consider the influence of Buoyancy-driven flow on it. Similarly, Buoyancy-driven flow also has an effect on turbulence. $G_{b}$ in (15) and (16) is the turbulence kinetic energy generated by Buoyancy given by 


$$
G_{b}=\beta g_{i} \frac{\mu_{t}}{\operatorname{Pr}_{t}} \frac{\partial T}{\partial x_{i}}
$$

where $g_{i}$ is the component of gravity in the direction of $i$, $\operatorname{Pr}_{t}=1 / \alpha$ is the Prandtl number of turbulent energy. $\alpha$ can be calculated from (17), where $\alpha_{0}=1 / \operatorname{Pr}=k / \mu c_{p}$, and $\beta$ is defined as

$$
\beta=-\frac{1}{\rho}\left(\frac{\partial \rho}{\partial T}\right)_{p}
$$

It can be seen from the $k$ equation that Buoyancy enhances turbulence kinetic energy in the unstable layer and suppresses turbulence in the stable layer. In the $\varepsilon$ equation, the effect of Buoyancy on turbulence is reflected in the constant $C_{3 \varepsilon}$, which is calculated by the following equation.

$$
C_{3 \varepsilon}=\tanh \left|\frac{v}{u}\right|
$$

where $v$ is the component of the fluid velocity in direction of gravity, and $u$ is the component of the fluid velocity in the direction perpendicular to the direction of gravity.

\section{Virtual model verification with experiment and simulation}

After the virtual model of blackbody cavity sensor for continuous temperature measurement of molten steel is established, the accuracy of the virtual model must be verified. Here, the accuracy of the virtual model is verified based on temperature measurement accuracy.

During simulation, the solution strategy for the influence of different nitrogen flow rates on temperature measurement accuracy includes two steps. Step 1. The temperature distribution of the outer wall of the blackbody cavity sensor is given first. Without blowing nitrogen, the simulation computation is performed until the internal temperature field of the blackbody cavity sensor reaches a steady state. Step 2. The nitrogen with different flow rates is blown into the cavity. The influence of different nitrogen flow rates on the temperature measurement accuracy is simulated with the virtual model.

In real-world experiment, two groups of sensors are inserted into the same tundish and measure simultaneously. The temperature measurement values of sensor $\mathrm{A}_{i}$ are the true molten steel temperature. Meanwhile, nitrogen with different flow rates are blown into sensor $\mathrm{B}_{i}$. Through analysing the differences between the temperature measurement values of the two groups of sensors, the influence of different nitrogen flow rates on the temperature measurement value is obtained.

\subsection{Boundary conditions}

Through real-world experiment of the sensor $A_{i}$, a temperature distribution of the cavity is selected, as shown in Table 1. The obtained temperature distribution is set as the initial condition of the simulation, that is, the initial temperature distribution of the outer wall of the sensor's outer tube is shown in Table 1.

Through the Step 1 of the solving strategy, the temperature distribution of the inner cavity can be simulated, which is consistent with Table 1.

Table 1. Initial temperature distribution of the outer wall of the outer tube.

\begin{tabular}{cc||cc}
\hline $\begin{array}{c}\text { Distance from } \\
\text { the target } \\
\text { surface } \mathrm{a}^{\mathrm{m}}(\mathrm{m})\end{array}$ & $\begin{array}{c}\text { Inner wall } \\
\text { temperature } \\
(\mathrm{K})\end{array}$ & $\begin{array}{c}\text { Distance from } \\
\text { the target } \\
\text { surface }(\mathrm{m})\end{array}$ & $\begin{array}{c}\text { Inner wall } \\
\text { temperature } \\
(\mathrm{K})\end{array}$ \\
\hline 0.164 & 1802.15 & 0.464 & 1459.11 \\
0.214 & 1802.15 & 0.514 & 1329.59 \\
0.264 & 1802.15 & 0.564 & 1299.23 \\
0.314 & 1802.15 & 0.614 & 1249.65 \\
0.364 & 1787.98 & 0.664 & 1208.16 \\
0.389 & 1786.97 & 0.714 & 1167.68 \\
0.414 & 1695.90 & 0.764 & 1103.93 \\
0.439 & 1558.28 & 0.814 & 1017.92 \\
\hline
\end{tabular}

${ }^{\text {a }}$ Target surface refers to the bottom end of the outer tube' inner wall.

\subsection{Material properties}

The verification for the temperature measurement accuracy is carried out when the temperature measurement value reaches a steady state under blowing nitrogen gas. In fact, soot particles and vapor inside the sensor cavity have been blown out. Therefore, when studying the temperature measurement accuracy of the sensor with different nitrogen flow rates, it is only necessary to set the properties of the material including fluid (nitrogen), outer tuber (Al-carbon), and inner tube (corundum), without setting the material properties of the soot particle and vapor. The properties of the three materials are fitted as the temperature-dependent functions $p=f(T)$ ( $p$ represents a certain property) [1516]. The specific physical parameters of the three materials, including density, specific heat, thermal conductivity, viscosity, absorption and scattering coefficient, are no longer given in details.

\subsection{Virtual model verification based on temperature measurement accuracy}

Table 2 shows the temperature values of sensor $\mathrm{A}_{i}$ and $\mathrm{B}_{i}$ after the temperature is stable. As mentioned above, sensor $A_{i}$ is a normal measurement without blowing nitrogen, and sensor $\mathrm{B}_{i}$ is measured with different nitrogen flow rates. It can be seen that as the flow rate of nitrogen increases, the temperature deviation increases.

Table 2. Temperature deviation of different nitrogen flow rates.

\begin{tabular}{cccc}
\hline $\begin{array}{c}\text { Nitrogen } \\
\text { flow rates } \\
\left(\mathrm{m}^{3} / \mathrm{h}\right)\end{array}$ & $\begin{array}{c}\text { Sensor } \mathrm{A}_{i} \\
\left({ }^{\circ} \mathrm{C}\right)\end{array}$ & $\begin{array}{c}\text { Sensor } \mathrm{B}_{i} \\
\left({ }^{\circ} \mathrm{C}\right)\end{array}$ & $\begin{array}{c}\text { Temperature } \\
\text { deviation }\left({ }^{\circ} \mathrm{C}\right)\end{array}$ \\
\hline 0.25 & 1528 & 1528 & 0 \\
0.5 & 1528 & 1528 & 0 \\
0.8 & 1525 & 1524 & 1 \\
1.0 & 1529 & 1528 & 1 \\
1.2 & 1522 & 1521 & 1 \\
1.5 & 1529 & 1527 & 2 \\
1.7 & 1524 & 1522 & 2 \\
2.0 & 1529 & 1524 & 5 \\
2.5 & 1529 & 1522 & 7 \\
\hline
\end{tabular}


At the same time, through simulation, the temperature distributions of the inner wall of the cavity under different nitrogen flow rates are obtained. The distance from the bottom end of the inner corundum tube to the target surface is $100 \mathrm{~mm}$. Therefore, it is only necessary to observe the temperature distribution at the inner wall of outer tube at 0 to $100 \mathrm{~mm}$. Fig. 4 shows the temperature distributions of the inner wall of outer tube under different nitrogen flow rates.

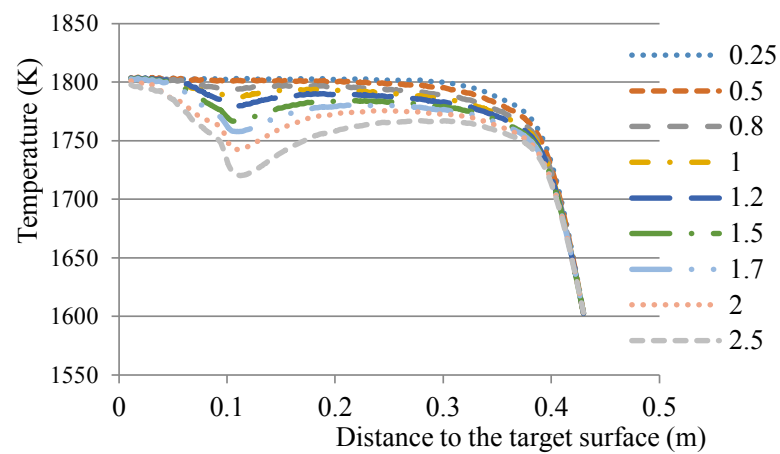

Fig. 4. Temperature distributions of the inner wall of outer tube under different nitrogen flow rates.

After obtaining the temperature distributions of different nitrogen flow rates, the emissivity of the whole cavity can be calculated according to the existing calculation method [1-3, 24], as shown in column 4 of Table 3. The method is as follows.

$$
E=\sigma T_{1}^{4}=\varepsilon \sigma T_{0}^{4}
$$

where $E$ is the received energy, $T_{1}$ is the radiant temperature, $\varepsilon$ is the emissivity of the whole cavity, $T_{0}$ is the true temperature of the target surface. The cavity emissivity $\varepsilon$ can be derived, which is a $T_{0}$ and $T_{1}$ dependent function, as

$$
\left(\frac{T_{1}}{T_{0}}\right)^{4}=\varepsilon
$$

Through derivation of $\varepsilon$ on both sides of (24), the following equation is obtained.

$$
4\left(\frac{T_{1}}{T_{0}}\right)^{3} \frac{d}{d \varepsilon}\left(\frac{T_{1}}{T_{0}}\right)=1
$$

The following equation can be derived from (25):

$$
d\left(T_{1}\right)=\frac{T_{0}}{4}\left(\frac{T_{0}}{T_{1}}\right)^{3} d \varepsilon
$$

Substituting (24) into (26), and using $\varepsilon$ and $T_{0}$ to replace $T_{1}$, the following equation can then be derived:

$$
d\left(T_{1}\right)=\frac{T_{0}}{4} \varepsilon^{\frac{3}{4}} d \varepsilon
$$

The temperature deviation can be calculated by substituting the cavity emissivity and target surface temperature in Table 3 into (27), as shown in column 5 of Table 3.

Comparing Table 2 to Table 3 , it can be found that the deviation between the temperature deviation obtained by the real-world experiment and the one obtained by the simulation calculation is at most $1.4^{\circ} \mathrm{C}$, which satisfies the target in this research. Therefore, it can be considered that the virtual model is accurate. The subsequent sensor structure design and parameter optimization, such as nitrogen flow rate and inner tube length, can be performed based on the verified virtual model.

Table 3. The emissivity of the whole cavity under different flow rate.

\begin{tabular}{ccccc}
\hline $\begin{array}{c}\text { Flow } \\
\text { rates } \\
\left(\mathrm{m}^{3} / \mathrm{h}\right)\end{array}$ & $\begin{array}{c}\text { Material } \\
\text { emissivity }\end{array}$ & $\begin{array}{c}\text { Target surface } \\
\text { temperature } \\
(\mathrm{K})\end{array}$ & $\begin{array}{c}\text { Cavity } \\
\text { emissivity }\end{array}$ & $\begin{array}{c}\text { Temperature } \\
\text { deviation } \\
\left({ }^{\circ} \mathrm{C}\right)\end{array}$ \\
\hline 0.25 & 0.7 & 1804.12 & 0.999261366 & 0.3 \\
0.5 & 0.7 & 1804.08 & 0.999122977 & 0.4 \\
0.8 & 0.7 & 1803.87 & 0.998502544 & 0.7 \\
1.0 & 0.7 & 1803.57 & 0.997683021 & 1.0 \\
1.2 & 0.7 & 1803.69 & 0.997919967 & 0.9 \\
1.5 & 0.7 & 1803.37 & 0.996959406 & 1.4 \\
1.7 & 0.7 & 1803.17 & 0.996349635 & 1.6 \\
2.0 & 0.7 & 1800.84 & 0.990217233 & 4.4 \\
2.5 & 0.7 & 1797.75 & 0.981344126 & 8.4 \\
\hline
\end{tabular}

\section{Conclusion and future works}

With regard to the actual requirement of the blackbody cavity sensor, this paper first redesigns the structure of the sensor, and establishes its virtual model, which has high fidelity with physical sensor and can truly reflect its behaviour, rule, constraint, etc. Then through the comparison between actual experiment and virtual model simulation, the accuracy of the virtual model is verified.

In the future, on one hand, more complete models might need to be constructed, which may cover multiphase flow, phase change, and soot particle radiation under transient conditions. On the other hand, the design improvement can be simulated based on the verified virtual model, such as the influence of different nitrogen flow rates and different inner tube's lengths on temperature measurement accuracy and temperature measurement stability time.

\section{References}

1. G. Mei, J. Zhang, X. Wang, S. Zhao, Z. Xie, Appl. Opt., 54(13), pp. 3948-3955 (2015)

2. G. Mei, J. Zhang, S. Zhao, Z. Xie, Appl. Opt., 53(11), pp. 2507-2514 (2014)

3. Z. Xie, Y. Ci, H. Meng, H. Zhang, Chinese Journal of Scientific Instrument, 26(5), pp. 446-448 (2005)

4. NIST-JANAF Thermochemical Tables. http://kinetic s.nist.gov/janaf.

5. N.P. Suh, Axiomatic Design - Advances and Applica tions (Oxford University Press, New York, 2009).

6. M.K. Thompson, CIRP Annals, 62(1), pp. 115-118 (2013)

7. O. Kulak, S. Cebi, C. Kahraman, Expert Syst. Appl. 37(9), pp. 6705-6717 (2010)

8. A. Liu, T.M. Li, Procedia CIRP, 53, pp. 119-124. (2016) 
9. J.W. Melvin, N.P. Suh, CIRP Annals, 51(1), pp. 107110 (2002)

10. S.C.Y. Lu, A. Liu, Proceedings of ICAD2011, The Sixth International Conference on Axiomatic Design ICAD-2011-01, Daejeon, Korea, 2011

11. A. Liu, S.C.Y. Lu, CIRP Annals, 64(1), pp. 153-156 (2015)

12. R.W. Hedrick, R.J. Urbanic, Comput-Aided Des. Applic. 10(4), pp. 567-583 (2013)

13. M.C. Leu, H.A. ElMaraghy, A.Y.C. Nee, S.K. Ong, M. Lanzetta, M. Putz, W. Zhu, A. Bernard, CIRP Anna. 62(2), pp. 799-822 (2013)

14. S.V. Patankar, Z. Zhang, Numerical calculation of heat transfer and fluid flow (Science Press, Beijing, 1984)

15. Y. Ma, R. Fang, Practical Thermophysical Properties Handbook (China Machine Press, Being, 1986)

16. J. Zhang, Handbook of Thermophysical Properties of Commonly Used Materials (New Times Press, Beijing, 1987)
17. W. Fiveland, A. Jamaluddin, J. Thermophys, 5(3), pp. 335-339 (1991)

18. G.D. Raithby, E.H. Chui, J. Heat Transfer. 112, pp. 415-423 (1990)

19. W.A. Fiveland, J. Thermophys Heat Transfer. 2, pp. 309-316 (1988)

20. E.H. Chui, G.D. Raithby, Numer. Heat Transfer, Part B. 23, pp. 269-288 (1993)

21. S.A. Orszag, V. Yakhot, W.S. Flannery, F. Boysan, D. Choudhury, J. Maruzewski, B. Patel, International Conference on Near-Wall Turbulent Flows, Tempe, Arizona (1993)

22. B.E. Launder, D.B. Spalding, Lectures in Mathematical Models of Turbulence (Academic Press, London, 1972)

23. V. Yakhot, S.A. Orszag, J. Sci. Comput. 1(1), pp. 151 (1986)

24. Y. Ci, Z. Xie, H. Zhang, J. Northeastern University (Natural Science), 25(5), pp. 460-462 (2004) 